\title{
Incidence of Respiratory Complications Following Lumbar Spine Surgery
}

\author{
RAJAN MURGAI, BS, ANTHONY D'ORO, BA, PATRICK HEINDEL, MD, KYLE SCHOELL, MD, KAKU \\ BARKOH, MD, ZORICA BUSER, PHD, JEFFREY C. WANG, MD \\ Department of Orthopaedic Surgery, Keck School of Medicine, University of Southern California, Los Angeles
}

\begin{abstract}
Background: The purpose of this study is to assess the incidence, risk factors for, and types of respiratory complications occurring in patients undergoing lumbar spine surgery.

Methods: Patients undergoing various lumbar spine surgeries from 2007 to 2014 were identified using the PearlDiver patient record database from the nationwide insurance provider Humana Inc. Patient records were analyzed using International Classification of Diseases, Ninth Revision codes and Current Procedural Terminology codes to determine the incidence of pneumonia, pleural effusion, pulmonary collapse, and acute respiratory failure for each procedure type. The incidence of these complications in patients with the risk factors diabetes mellitus, chronic obstructive pulmonary disease (COPD), and smoking was also examined.

Results: A total of 64,891 patients (33,280 females; 31,611 males) within the Humana database underwent various lumbar procedures from 2007 to 2014. The overall incidence of respiratory complications in patients undergoing lumbar procedures was $5.7 \%(\mathrm{n}=3694)$ within 1 month of having the procedure. Pulmonary collapse was the most common complication with an incidence of $4.3 \%(\mathrm{n}=2792)$, followed by pneumonia $1.98 \%(\mathrm{n}=1285)$, acute respiratory failure $1.97 \%(\mathrm{n}=1279)$, and pleural effusion $1.6 \%(\mathrm{n}=1048)$. For each respiratory complication studied, single level discectomy had the lowest complication rate and multilevel anterior lumbar interbody fusion had the highest complication rate. The incidence of each individual respiratory complication was higher in patients who had a history of smoking, COPD, or diabetes mellitus than it was in patients with none of these 3 risk factors $(P<.01)$.

Conclusion: The results of this study show that patients who have a history of smoking, COPD, or diabetes mellitus are at a greater risk for respiratory complications following lumbar spine surgery. These findings are useful for patient selection, clinical decision-making, and preoperative counseling.
\end{abstract}

Lumbar Spine

Keywords: respiratory complications, lumbar spine surgery, chronic obstructive pulmonary disease, diabetes mellitus, postoperative complications, smoking, pulmonary complications

\section{INTRODUCTION}

Respiratory complications following surgery are a major cause of increased length of stay and perioperative morbidity and mortality. ${ }^{1-3}$ Approximately 1 in 5 patients who develop postoperative respiratory failure die within 30 days. ${ }^{3}$ Furthermore, pulmonary complications may be more likely than cardiac complications to predict long-term mortality after surgery, especially among older patients. ${ }^{4}$ The most morbid postoperative respiratory complications include pneumonia, respiratory failure, atelectasis, and exacerbation of underlying chronic lung disease. Respiratory complications are more common after surgeries with extended surgical time, abdominal surgery, and thoracic surgery. ${ }^{2}$ Lumbar spinal surgeries can be lengthy and involve both posterior and anterior approaches, placing patients at a high risk for these complications.

Furthermore, some patient characteristics put them at greater risk for respiratory complications following surgery. Recent research has established several factors that lead to increased respiratory complications following general surgical procedures. Qaseem et $\mathrm{al}^{2}$ reported that chronic obstructive pulmonary disease (COPD) was the most commonly identified risk factor for postoperative pulmonary complications (odds ratio 1.79). Additionally, diabetes mellitus and smoking have been identified as risk factors for postoperative lung injury following noncardiothoracic surgery. ${ }^{2,5}$ Older age is also an established risk factor for respiratory complications: 70 to 79 odds ratio 3.04 and 60 to 69 odds ratio 2.09 when compared to patients $<60$ 
years of age. ${ }^{2}$ As the population ages, surgeons will need to treat increasingly elderly patients. Other risk factors found to have a significant effect on the incidence of respiratory complications are congestive heart failure, functional dependence, and American Society of Anesthesiologists (ASA) class. ${ }^{2}$ While recent research has evaluated respiratory complications following surgery, most of these studies focused on patients undergoing general surgical procedures. Few studies have focused on orthopedic, spine, or lumbar procedures. Among the few studies examining this topic, varying rates of complications were observed and the definition of what constitutes a complication varied. Further, several studies were limited by examining pulmonary complications as an aggregate or by examining the complication rates of only a select type of lumbar procedure. ${ }^{6-10}$ The purpose of this study was to more accurately assess the incidence, risk factors for, and types of respiratory complications occurring in patients undergoing the 5 most common lumbar spine surgical procedures. Understanding the likelihood of respiratory complications and identifying risk factors for them can allow for evidence-based decisions regarding patient selection, efforts to prevent pulmonary complications from occurring in those who are at risk, and improved preoperative counseling.

\section{MATERIALS AND METHODS}

A search of 19907868 patient records compiled from the nationwide insurance provider Humana Inc. was conducted using the commercially available PearlDiver software. Patient records were searched using International Classification of Diseases, Ninth Revision (ICD-9) codes and current procedural terminology (CPT) codes to isolate patients undergoing single and multilevel fusion, laminectomy, and discectomy from January 2007 to December 2014. For this type of study formal consent is not required. Demographic information including age and sex was collected. The fusion procedures investigated were anterior lumbar interbody fusion (ALIF), posterior lumbar interbody fusion (PLIF), and posterolateral lumbar fusion (PLF). Patients receiving oblique lateral interbody fusion (OLIF) were included in the ALIF group and patients receiving transforaminal interbody fusion (TLIF) were included in the PLIF group. Patients who received more than 1 type of fusion concurrently were included in both groups. The discectomy group included patients who underwent a laminotomy with decompression of nerve roots and excision of a herniated intervertebral disc at 1 or more interspace. The laminectomy group included patients who underwent decompression of the spinal cord and/ or cauda equina with laminectomy, vertebral corpectomy, or transpedicular decompression. Concomitant fusion was excluded for patients in the discectomy and laminectomy groups. Any patients with previous lumbar surgeries were excluded. Further, patients with a history of previous spine trauma, tuberculosis, primary or metastatic bone neoplasm, osteomyelitis, discitis, or surgical site infection were excluded from the study.

Patient records were subsequently analyzed to determine complication rates for each procedure type. The specific categories of respiratory complications studied were pneumonia, pleural effusion, pulmonary collapse (atelectasis), and acute respiratory failure. The risk factors studied were smoking, diabetes mellitus, and COPD. Each complication category was split into 5 cohorts: (1) patients without a history of diabetes mellitus, COPD, or smoking; (2) patients with diabetes mellitus; (3) patients with COPD; (4) patients with a history of smoking; and (5) patients with all 3 risk factors (diabetes, COPD, smoking). Complication rates were analyzed at 1 month postoperatively. The ICD-9 diagnosis codes that comprise each category of respiratory complication and risk factor can be found in Table 1. The study design is outlined in Figure 1.

\section{Statistical Methods}

For each complication, Pearson $\chi^{2}$ analyses were run on unpaired data output values for each procedure type to evaluate the observed differences between complication rates at 1 month. Further, Pearson $\chi^{2}$ analyses were run to evaluate the differences in complication rates observed between single level and multilevel fusion. The relative risk ratios (RR) and associated $95 \%$ confidence intervals were determined for each risk factor to evaluate the observed differences in their complication rates.

\section{RESULTS}

The search returned information on 64891 patients who had 1 of the studied lumbar procedures from 2007 to 2014. Of these patients, 51.3\% $(\mathrm{n}=33280)$ were female and $48.7 \%(\mathrm{n}=31611)$ 
Table 1. ICD-9 and CPT codes used for inclusion and exclusion.

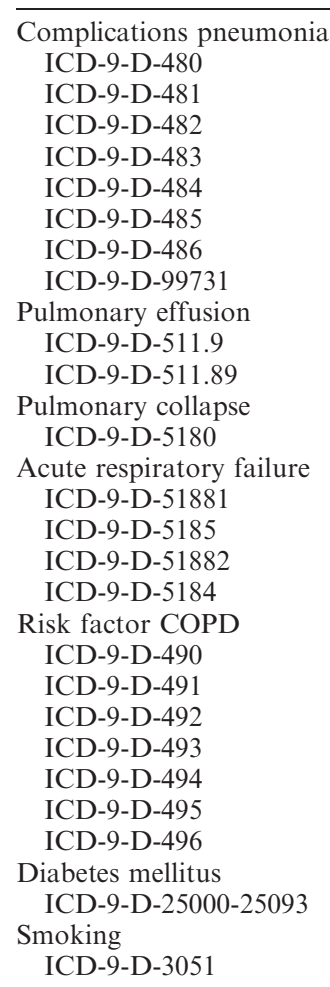

Abbreviations: ICD-9, International Classification of Diseases, Ninth Revision CPT, current procedural terminology.

were male. The age group with the highest percentage of patients was 65 to 69 with $21.7 \%$ (n $=14$ 069) of all studied patients, followed by the 70 to $75(19.3 \%, \mathrm{n}=12523)$ and 75 to $80(12.2 \%, \mathrm{n}=$ 7907) age groups. The most common procedure performed was laminectomy $(\mathrm{n}=21788)$ and least common was multiple level ALIF $(\mathrm{n}=1843)$.

The overall incidence of respiratory complications in patients undergoing lumbar procedures was $5.7 \% \quad(\mathrm{n}=3694)$ within 1 month of having the procedure. Of these complications, pulmonary collapse was the most common at $4.3 \%(n=2792)$ followed by pneumonia $1.98 \%(\mathrm{n}=1285)$, acute respiratory failure $1.97 \%(\mathrm{n}=1279)$, and pleural effusion $1.6 \% \quad(\mathrm{n}=1048)$. For each respiratory complication studied, discectomy had the lowest complication rate and ALIF had the highest complication rate. Also, there was a statistically significant difference between the complication rates of PLIF, ALIF, PLF, discectomy, and laminectomy $(P<.001$; Table 2$)$ for each respiratory complication. Further, single level fusion had a lower complication rate than multilevel fusion $(P<.001$; Table 2).

Overall, the incidence of pulmonary collapse was $6.00 \%(\mathrm{RR}=1.75)$ in patients with all 3 risk factors,

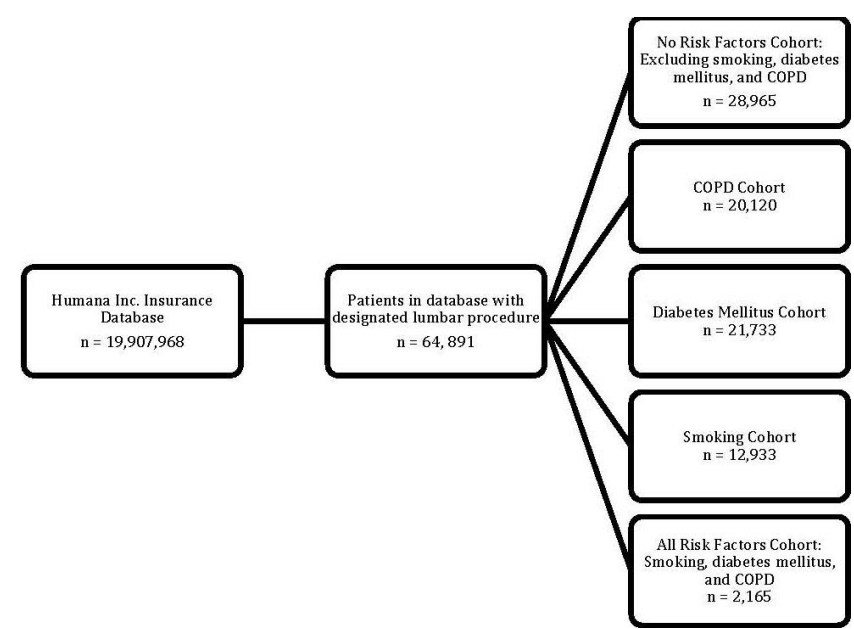

Figure 1. Flowchart of patient selection and complication analysis.

$5.60 \%(\mathrm{RR}=1.64)$ in patients with $\mathrm{COPD}, 5.01 \%$ $(\mathrm{RR}=1.46)$ in patients with diabetes, $4.05 \%(\mathrm{RR}=$ $1.18)$ in patients with a history of smoking, and $3.42 \%$ in patients without risk factors $(P<.05$; Table 3). The incidence of pneumonia was highest in patients with all 3 risk factors $4.34 \%(\mathrm{RR}=3.22)$, followed by patients with COPD 3.54\% (RR = $2.63)$, patients with diabetes $2.73 \%(\mathrm{RR}=2.03)$, patients with a history of smoking $2.40 \%(\mathrm{RR}=$ 1.78), and patients without risk factors $1.35 \%(P<$ .05 ; Table 3$)$. The incidence of acute respiratory failure was $4.30 \%(\mathrm{RR}=3.13)$ in patients with all 3 risk factors, $3.43 \%(R R=2.50)$ in patients with COPD, $3.02 \%(R R=2.20)$ in patients with diabetes, $2.20 \%(\mathrm{RR}=1.6)$ in patients with a history of smoking, and $1.37 \%$ in patients without risk factors $(P<.05$; Table 3$)$. The rate of pleural effusion was highest in patients with all 3 risk factors $2.82 \%$ (RR $=2.20$ ), followed by patients with COPD $2.55 \%$ $(\mathrm{RR}=1.99)$, patients with diabetes $2.08 \%(\mathrm{RR}=$ 1.62 ), patients with a history of smoking $1.62 \%$ (RR $=1.26)$, and patients without any risk factor $1.28 \%$ $(P<.05$; Table 3).

\section{DISCUSSION}

The results of our study show that patients with a history of COPD, diabetes mellitus, or smoking are at greater risk of developing all of the studied pulmonary complications. Patients undergoing fusion procedures have higher respiratory complication rates than those undergoing discectomies or laminectomies. Furthermore, patients undergoing multilevel fusion have greater respiratory complication rates than those undergoing single level fusion. 
Table 2. Incidence of complications by procedure type.

\begin{tabular}{|c|c|c|c|}
\hline Complication & Procedure & $\begin{array}{c}\text { Rate of } \\
\text { Complication, \% }\end{array}$ & $P$-Value \\
\hline \multirow[t]{7}{*}{ Pneumonia } & PLIF any level & 2.86 & \multirow[t]{5}{*}{$<.001$} \\
\hline & ALIF any level & 3.38 & \\
\hline & PLF any level & 2.89 & \\
\hline & Discectomy any level & 0.79 & \\
\hline & Laminectomy & 1.57 & \\
\hline & Single level fusion & 2.58 & \multirow[t]{2}{*}{$<.001$} \\
\hline & Multilevel fusion & 3.41 & \\
\hline \multirow{7}{*}{$\begin{array}{l}\text { Acute respiratory } \\
\text { failure }\end{array}$} & PLIF any level & 2.94 & \multirow[t]{5}{*}{$<.001$} \\
\hline & ALIF any level & 4.51 & \\
\hline & PLF any level & 2.99 & \\
\hline & Discectomy any level & 0.55 & \\
\hline & Laminectomy & 1.29 & \\
\hline & Single level fusion & 2.28 & \multirow[t]{2}{*}{$<.001$} \\
\hline & Multilevel fusion & 4.28 & \\
\hline \multirow[t]{7}{*}{ Pulmonary collapse } & PLIF any level & 6.66 & \multirow[t]{5}{*}{$<.001$} \\
\hline & ALIF any level & 9.62 & \\
\hline & PLF any level & 7.16 & \\
\hline & Discectomy any level & 1.22 & \\
\hline & Laminectomy & 2.59 & \\
\hline & Single level fusion & 5.62 & \multirow[t]{2}{*}{$<.001$} \\
\hline & Multilevel fusion & 9.37 & \\
\hline \multirow[t]{7}{*}{ Pleural effusion } & PLIF any level & 2.19 & \multirow[t]{5}{*}{$<.001$} \\
\hline & ALIF any level & 3.45 & \\
\hline & PLF any level & 2.56 & \\
\hline & Discectomy any level & 0.43 & \\
\hline & Laminectomy & 1.07 & \\
\hline & Single level fusion & 1.84 & \multirow[t]{2}{*}{$<.001$} \\
\hline & Multilevel fusion & 3.41 & \\
\hline
\end{tabular}

Abbreviations: PLIF, posterior lumbar interbody fusion; ALIF, anterior lumbar interbody fusion; PLF, posterolateral lumbar fusion.

The overall incidence of respiratory complications in patients undergoing lumbar procedures was $5.7 \%$ within 1 month of having the procedure. Varying rates of respiratory complications were observed in the few other studies examining respiratory complications following spine surgery. A retrospective chart review conducted by JulesElysee et $\mathrm{al}^{6}$ in 2004 on pulmonary complications following anterior-posterior thoracic lumbar fusions reported much higher complication rates. This study reported the following complication rates: $53 \%$ atelectasis, $21 \%$ infiltrates, $13 \%$ partial or complete lobar collapse, and 3\% pneumonia. However, the outcome measure of this study was radiographic abnormalities, which do not always have clinical significance. Further, this study was limited by a small sample size $(n=59)$ and included patients with thoracic spinal procedures who were more likely to have invasion of the thoracic cavity and increased pulmonary complication rates. ${ }^{6} \mathrm{Fu}$ et $\mathrm{al}^{8}$ reported the rate of respiratory complications/pulmonary embolus to be $0.2 \%$ following the surgical treatment of lumbar stenosis. However, this study was conducted on patients in the Scoliosis Research Society morbidity and mortality database, which is based upon self-reporting. Thus, it may underesti-
Table 3. Incidence of complications by risk factor.

\begin{tabular}{lllll}
\hline Complication & $\begin{array}{c}\text { Risk } \\
\text { Factor }\end{array}$ & $\begin{array}{c}\text { Rate of } \\
\text { Complication, \% }\end{array}$ & RR & 95\% CI \\
\hline Pneumonia & None & 1.35 & Ref. & \\
& COPD & 3.54 & 2.63 & $2.33-2.97$ \\
& Diabetes & 2.73 & 2.03 & $1.79-2.30$ \\
Acute respiratory & Smoking & 2.40 & 1.78 & $1.54-2.06$ \\
failure & All & 4.34 & 3.22 & $2.59-4.02$ \\
& None & 1.37 & Ref. & \\
& COPD & 3.43 & 2.50 & $2.21-2.82$ \\
Pulmonary collapse & Diabetes & 3.02 & 2.20 & $1.94-2.48$ \\
& Smoking & 2.20 & 1.60 & $1.38-1.86$ \\
& All & 4.30 & 3.13 & $2.51-3.90$ \\
& None & 3.42 & Ref. & \\
COPD & 5.60 & 1.64 & $1.50-1.78$ \\
& Diabetes & 5.01 & 1.46 & $1.34-1.59$ \\
& Smoking & 4.05 & 1.18 & $1.07-1.31$ \\
& All & 6.00 & 1.75 & $1.47-2.09$ \\
& None & 1.28 & Ref. & \\
& COPD & 2.55 & 1.99 & $1.74-2.27$ \\
& Diabetes & 2.08 & 1.62 & $1.42-1.86$ \\
& Smoking & 1.62 & 1.26 & $1.07-1.49$ \\
& All & 2.82 & 2.20 & $1.68-2.87$ \\
\hline
\end{tabular}

Abbreviation: All, all 3 risk factors (chronic obstructive pulmonary disease [COPD], diabetes, smoking); RR, risk ratio.

mate the true complication rates. ${ }^{8}$ Kalanithi et $\mathrm{al}^{9}$ reported a pulmonary complication rate of $2.6 \%$, but this study was limited to posterior lumbar fusion for acquired spondylolisthesis. The results from our study were in accordance with a study conducted by Lee et $\mathrm{al}^{10}$ who reported a pulmonary complication rate of $7 \%$ following lumbar spine surgery. This study also found diabetes, COPD, and surgical invasiveness to be significant risk factors for respiratory complications following lumbar spine surgery. ${ }^{10}$ However, while this study examined the overall pulmonary complication rate following lumbar spine surgery solely as an aggregate, our study examined specific pulmonary complications following each of the 5 most common lumbar spine surgeries.

The most common complication, pulmonary collapse, occurred in $4.3 \%$ of patients overall. Pulmonary collapse is a significant complication because it is the core mechanism responsible for postoperative hypoxemic events in the majority of patients after surgery. ${ }^{11}$ Functional residual capacity is reduced following abdominal and thoracic surgery in a predictable pattern due to incisional pain and reflex dysfunction of the diaphragm. Forced vital capacity is known to decrease by $50 \%$ from baseline and return to normal in 1 to 2 weeks in patients following upper abdominal surgery. ${ }^{12}$ Decreased functional residual capacity and forced vital capacity leads to atelectasis. ${ }^{13}$ Furthermore, general anesthesia promotes atelectasis. ${ }^{11}$ Jules- 
Elysee et $\mathrm{al}^{6}$ reported atelectasis in $53 \%$ of patients undergoing anterior-posterior thoracic lumbar fusions. The higher incidence of pulmonary collapse in this study may be attributed to the primary outcome measure being radiographic abnormalities and the inclusion of thoracic level fusions. Atelectasis detected on radiographs is not always clinically significant and often does not change management. Thus, it is less likely to be consistently coded for.

Acute respiratory failure was observed in $1.97 \%$ of patients in our study. A retrospective cohort study at a regional level-1 trauma center reported respiratory failure in $13.5 \%$ of patients following surgically treated thoracic and lumbar spine fractures. ${ }^{14}$ However, many of these patients had other associated injuries and the study notes that thoracic level injury increased risk for respiratory failure. Additionally, the highest rates of respiratory failure after spine surgery have been observed in trauma patients, which were excluded from our study. Another retrospective study of patients with traumatic fractures of the lumbar spine reported acute respiratory distress syndrome in $3.6 \%$ of patients. ${ }^{15}$ The incidence of acute respiratory failure was significantly higher in patients with a history of COPD, diabetes, or smoking than it was in patients without any risk factors. Diabetes, smoking, and COPD have been shown to be strong predictors of postoperative lung injury in studies of patients undergoing general surgery. ${ }^{2,5}$

Our study demonstrated that the incidence of pneumonia was significantly higher in patients with COPD, diabetes, and smoking than patients without these risk factors. In agreement with our study, Bohl et $\mathrm{al}^{7}$ found COPD, both noninsulin dependent diabetes mellitus and insulin diabetes mellitus, and greater number of operative levels to be independent risk factors for the development of pneumonia following posterior lumbar fusion. However, their study did not find current smokers to be at a significantly increased risk for postoperative pneumonia. ${ }^{7}$ It is established that patients with COPD are at increased risk for developing communityacquired pneumonia. ${ }^{16}$ It is thought that this increased risk of developing pneumonia may be associated with airflow limitation, the presence of pulmonary emphysema, and inhaled corticosteroid use in patients with COPD. ${ }^{16,17}$ In a meta-analysis, Smetana $^{3}$ established pre-existing COPD as a risk factor for postoperative pneumonia. The diabetic lung is more susceptible to infection and more likely to host severe episodes of pneumonia. ${ }^{18}$ Additionally, diabetic patients have longer lengths of stay and higher readmission rates after lumbar spine surgery. ${ }^{19}$ Increased length of hospital stay is a risk factor for hospital-acquired pneumonia. ${ }^{20}$ The overall incidence of pneumonia was $1.98 \%$. The incidence of pneumonia was $2.89 \%$ in patients with PLF. Bohl et $\mathrm{al}^{7}$ found the incidence of pneumonia to be $.59 \%$ in a retrospective study of 12,428 patients undergoing posterior lumbar fusion. In their study, patients had to meet both strict radiologic criteria and signs/symptoms/laboratory criteria in order to be diagnosed with postoperative pneumonia, whereas our study relied on provider diagnosis coding.

$\chi^{2}$ statistical analysis showed significantly higher respiratory complication rates for ALIF than PLIF or PLF for all respiratory complications other than pneumonia $(P<.001)$. For all respiratory complications, patients undergoing fusion had significantly higher complication rates than those undergoing discectomy or laminectomy $(P<.001)$. Furthermore, patients undergoing multilevel fusion had higher respiratory complication rates than those undergoing single level fusion $(P<.001)$. This may be due to the increased operative time of multilevel fusion. ${ }^{21}$ Increased operative time and subsequent prolonged exposure to general anesthesia may increase risk for postoperative respiratory complications. ${ }^{11}$ Additionally, multilevel procedures tend to be more invasive than single level procedures. Lee et $\mathrm{al}^{10}$ found increased surgical invasiveness to be the largest risk factor for respiratory complications following lumbar spine surgery.

In our study, smoking was a significant risk factor for all of the studied pulmonary complications. In a study of perioperative pulmonary complications following combined anterior-posterior thoracic lumbar fusions, Jules-Elysee et $\mathrm{al}^{6}$ did not find smoking to be a significant risk factor in the development of pulmonary complications. Additionally, Bohl et $\mathrm{al}^{7}$ found that current smokers were not at a significantly increased risk for the development of pneumonia following posterior lumbar fusion procedures. However, smoking is an established risk factor for the development of postoperative medical complications after adult spinal deformity surgery. ${ }^{22}$ Smoking has also been shown to be a significant risk factor for postoperative pulmonary complications in patients undergoing major surgery. ${ }^{2,23}$ Schmid et $\mathrm{al}^{23}$ found the effect of smoking on postoperative pulmonary 
complications to be procedure dependent. Furthermore, their study found that current smokers had the highest incidence of respiratory complications, followed by former smokers and nonsmokers. ${ }^{23}$ This suggests that smoking cessation may help reduce the incidence of perioperative respiratory complications.

Preventive strategies may be employed to protect against postoperative pulmonary complications in those at increased risk. A recent review found that preoperative inspiratory muscle training was associated with a reduction in postoperative atelectasis, pneumonia, and duration of hospital stay. ${ }^{24}$ Preoperative smoking cessation, nutritional supplementation, and chest physical therapy have also been recommended to decrease postoperative pulmonary complications. Postoperative interventions to decrease the incidence of pulmonary complications in mechanically ventilated patients include chest physical therapy, intermittent suction, bronchodilators, positive end expiratory pressure valves, and inhaled mucolytics. ${ }^{25}$ Postoperative use of an incentive spirometer, careful control of pain that interferes with deep breathing and coughing, and intermittent positive pressure breathing have been recommended for the prevention of postoperative pneumonia in patients at increased risk. ${ }^{26}$ Intraoperative mechanical ventilation with low tidal volumes has also been shown to reduce the incidence of postoperative pulmonary complications. ${ }^{27}$

While the size and scope of our sample allow for improved understanding of the relationship between various risk factors and respiratory complications following lumbar spine surgery, it is limited by the fact that billing records are used as the source for our data. The potential for missing data exists due to the varying extent to which individual surgeons document diagnoses. Also, the clinical significance of some documented complications is unknown. Specifically, atelectasis is often a benign complication that many patients have postoperatively. Furthermore, unmeasured confounding and misclassification bias may be present in the data due the fact that diagnosis codes are sometimes vague. Since the Humana database was used for patient selection, it is possible there may be a selection bias due to the types of patients that the insurance provider insures. However, the database includes patients with individual commercial, statebased Medicaid, and Medicare insurance. Despite these limitations, using records from an insurance database was able to provide a geographically diverse sample that allows for reporting of national hospital complication rates that are representative of routine clinical practice. Furthermore, the large sample size offered by this method allows for comparing complication rates with greater statistical power. Another potential limitation of this study was that pulmonary complications were only studied at the 1-month mark. However, the impact of this limitation on pneumonia complication rates was probably minimal as Bohl et $\mathrm{al}^{7}$ showed that the incidence of postoperative pneumonia plateaus before the 30th postoperative day.

By using records from a large nationwide insurance database, our study was able to successfully provide the incidence of individual pulmonary complications following the 5 most common lumbar spine surgeries. This approach also demonstrated that COPD, diabetes, and smoking are significant risk factors for all studied respiratory complications following lumbar spine surgery. Furthermore, patients with all 3 risk factors had a higher complication rate than patients with no risk factors or any of the individual risk factors. Future studies are needed to determine if the severity of these comorbidities impacts the risk of postoperative respiratory complications. We believe that our findings are useful for physicians and patients considering lumbar spine surgery and allow for improved patient selection, counseling, and clinical decision-making.

\section{ACKNOWLEDGMENTS}

For part of the study, PearlDiver access was supported by departmental funds. This study did not require an IRB approval; all of the information was de-identified - we just had counts; no single patient numbers.

\section{REFERENCES}

1. Lawrence VA, Cornell JE, Smetana GW; American College of Physicians. Strategies to reduce postoperative pulmonary complications after noncardiothoracic surgery: systematic review for the American College of Physicians. Ann Intern Med. 2006;144(8):596-608.

2. Qaseem A, Snow V, Fitterman N, et al; Clinical Efficacy Assessment Subcommittee of the American College of Physicians. Risk assessment for and strategies to reduce perioperative pulmonary complications for patients undergoing noncardiothoracic surgery: a guideline from the American College of Physicians. Ann Intern Med. 2006;144(8):575-580.

3. Smetana GW. Preoperative pulmonary evaluation: identifying and reducing risks for pulmonary complications. Cleve Clin J Med. 2006;73(Suppl 1):S36-S41.

4. Manku K, Bacchetti P, Leung JM. Prognostic significance 
of postoperative in-hospital complications in elderly patients. I. Long-term survival. Anesth Analg. 2003;96(2):583-589.

5. Kelkar KV. Post-operative pulmonary complications after non-cardiothoracic surgery. Indian J Anaesth. 2015;59(9):599605.

6. Jules-Elysee K, Urban MK, Urquhart BL, Susman MH, Brown AC, Kelsey WT. Pulmonary complications in anteriorposterior thoracic lumbar fusions. Spine J. 2004;4(3):312-316.

7. Bohl DD, Mayo BC, Massel DH, et al. Incidence and risk factors for pneumonia after posterior lumbar fusion procedures: an ACS-NSQIP Study. Spine (Phila Pa 1976). 2016;41(12):1058-1063.

8. Fu KM, Smith JS, Polly DW Jr, et al. Morbidity and mortality in the surgical treatment of 10,329 adults with degenerative lumbar stenosis. $J$ Neurosurg Spine. 2010;12(5):443-446.

9. Kalanithi PS, Patil CG, Boakye M. National complication rates and disposition after posterior lumbar fusion for acquired spondylolisthesis. Spine (Phila Pa 1976). 2009;34(18):19631969.

10. Lee MJ, Hacquebord J, Varshney A, et al. Risk factors for medical complication after lumbar spine surgery: a multivariate analysis of 767 patients. Spine (Phila Pa 1976). 2011;36(21):1801-1806.

11. Karcz M, Papadakos PJ. Respiratory complications in the postanesthesia care unit: a review of pathophysiological mechanisms. Can J Respir Ther. 2013;49(4):21-29.

12. Latimer RG, Dickman M, Day WC, Gunn ML, Schmidt CD. Ventilatory patterns and pulmonary complications after upper abdominal surgery determined by preoperative and postoperative computerized spirometry and blood gas analysis. Am J Surg. 1971;122(5):622-632.

13. Wahba RW. Perioperative functional residual capacity. Can J Anaesth. 1991;38(3):384-400.

14. McHenry TP, Mirza SK, Wang J, et al. Risk factors for respiratory failure following operative stabilization of thoracic and lumbar spine fractures. $J$ Bone Joint Surg Am. 2006;88(5):997-1005.

15. Winkler EA, Yue JK, Birk $\mathrm{H}$, et al. Perioperative morbidity and mortality after lumbar trauma in the elderly. Neurosurg Focus. 2015;39(4):E2.

16. Eom JS, Song WJ, Yoo H, et al. Chronic obstructive pulmonary disease severity is associated with severe pneumonia. Ann Thorac Med. 2015;10(2):105-111.

17. Festic E, Bansal V, Gupta E, Scanlon PD. Association of inhaled corticosteroids with incident pneumonia and mortality in COPD patients; systematic review and meta-analysis. COPD. 2016;13(3):312-326.

18. Ardigo D, Valtuena S, Zavaroni I, Baroni MC, Delsignore R. Pulmonary complications in diabetes mellitus: the role of glycemic control. Curr Drug Targets Inflamm Allergy. 2004;3(4):455-458.

19. Guzman JZ, Iatridis JC, Skovrlj B, et al. Outcomes and complications of diabetes mellitus on patients undergoing degenerative lumbar spine surgery. Spine (Phila Pa 1976). 2014;39(19):1596-1604.

20. Lynch JP 3rd. Hospital-acquired pneumonia: risk factors, microbiology, and treatment. Chest. 2001;119(suppl 2):373S-384S.

21. Mirza SK, Deyo RA, Heagerty PJ, et al. Development of an index to characterize the "invasiveness" of spine surgery: validation by comparison to blood loss and operative time. Spine (Phila Pa 1976). 2008;33(24):2651-2661.

22. Soroceanu A, Burton DC, Oren JH, et al. Medical complications after adult spinal deformity surgery: incidence, risk factors, and clinical impact. Spine (Phila Pa 1976). 2016;41(22):1718-1723.

23. Schmid M, Sood A, Campbell L, et al. Impact of smoking on perioperative outcomes after major surgery. Am J Surg. 2015;210(2):221-229.

24. Katsura M, Kuriyama A, Takeshima T, Fukuhara S, Furukawa TA. Preoperative inspiratory muscle training for postoperative pulmonary complications in adults undergoing cardiac and major abdominal surgery. Cochrane Database Syst Rev. 2015;10.

25. Shander A, Fleisher LA, Barie PS, Bigatello LM, Sladen RN, Watson CB. Clinical and economic burden of postoperative pulmonary complications: patient safety summit on definition, risk-reducing interventions, and preventive strategies. Crit Care Med. 2011;39(9):2163-2172.

26. Centers for Disease Control and Prevention. Guidelines for prevention of nosocomial pneumonia. MMWR Recomm Rep. 1997;46(RR-1):1-79.

27. Güldner A, Kiss T, Serpa Neto A, et al. Intraoperative protective mechanical ventilation for prevention of postoperative pulmonary complications: a comprehensive review of the role of tidal volume, positive end-expiratory pressure, and lung recruitment maneuvers. Anesthesiology. 2015;123(3):692-713.

Disclosures and COI: There are no conflicts of interest for the current study. Disclosures outside of submitted work: ZB: consultancy: Xenco Medical, AO Spine; research support: SeaSpine (paid directly to institution). JCW: royalties: Biomet, Seaspine, Amedica, Synthes; investments/options: Fziomed, Nexgen, Vertiflex, Electrocore, Surgitech, Expanding Orthopedics, Osprey, Bone Biologics, Pearldiver; Board of Directors: North American Spine Society, North American Spine Foundation, AO Foundation, Cervical Spine Research Society; Editorial Boards: Spine, JAAOS, The Spine Journal, Clinical Spine Surgery, Global Spine Journal; fellowship funding (paid to institution): AO Foundation.

Corresponding Author: Zorica Buser, $\mathrm{PhD}$, Department of Orthopaedics, Keck School of Medicine, University of Southern California, 1450 San Pablo St., HC4 - \#5400A, Los Angeles, CA 90033. Phone: (323) 442-0206; Email: zbuser@usc. edu.

Published 21 December 2018

This manuscript is generously published free of charge by ISASS, the International Society for the Advancement of Spine Surgery. Copyright @ 2018 ISASS. To see more or order reprints or permissions, see http://ijssurgery.com. 